\title{
Research on the Knowledge Management Based on GIS
}

\author{
Minghai Luo \\ Wuhan Geotechnical Engineering and Surveying Institute \\ Wuhan, China
}

\begin{abstract}
Knowledge management is to collect, organize, process and transmit the information and provide effective means to make use of the information fully. The development and application of GIS and internet technology will import the bran-new concept for the knowledge management, promote the knowledge management to the direction of videotext and collaboration, and increase the longitudinal deepness and the transverse extent for the knowledge management. The development of knowledge management demands to build geographic information-based comprehensive resource system, carry out association and sharing of comprehensive information of multiple types and levels, and improve the knowledge management and knowledge finding capability of spatial information.
\end{abstract}

\section{Keywords-Knowledge management; Geographical information system; Internet}

\section{Introduction}

Knowledge management is the effective means to collect, process, manage, transmit and make use of information in this information explosion age. The main characteristics of knowledge economy is to take knowledge as the most important resource of economy, basic factor of production, and the main power of development. Information is just the flowing blood in the world economy network.

The development and application of geographical information system (GIS) will give the bran-new concept for knowledge management, which will expand the deepness and extent for the management, mining and using of spatial information, and will form the cognition map, network map and national conditions map related to geographical location.

Section one indicates the necessity of this research.

Section two introduces the concept of knowledge and knowledge management.

Section three introduces the concept and function of GIS.

Section four analyses the developing tendency of knowledge management based on GIS.

Section five summarizes the meaning of GIS application for knowledge management.

\section{Knowledge and Knowledge Management}

\section{A Data, Information and Knowledge}

In information society it is more important to possess information resource than to have natural resource. But information is not equal to knowledge. Frequently we urgently need information on the one hand, but a great deal of information idle aside and become out-dated and useless on the other hand. "We are flooded by information but suffer from the lack of knowledge.” [1]

Data, information and knowledge are the three closely related but different concepts. Data is the objective description of fact, such as figure, letter, symbol, image, sound and taste, which may be transacted and exchanged by hand or automation device. Information is the explanation of data, which is structured data. Data transaction is to explain data, and data becomes information only after explanation. Knowledge is the useful information, which may change people and things, and come from the collection, organization, processing, communication and utilization of information.

\section{B Knowledge Discovery and Knowledge Management}

Knowledge discovery originates from artificial intelligence and machine study, which may take out the connotative, unknown and potential information from data by concise manner. It's the data search process and do not need to put forward the hypothesis and question in advance.

Knowledge management is to combine the data processing capacity of information technology with the people's invention and innovation capacity, and transform the information to knowledge. The knowledge extraction technology is built on the basis of dada mining, which includes semantic, cooperative and visual means. ${ }^{[2]}$

\section{Development Phases and Direction of Knowledge Management}

Traditional information management went through five phases: in nineteen fifties, combination with documents and technology; in nineteen sixties, focus on the research of information processing technology to improve information service efficiency; in nineteen seventies, focus on the research of information processing automation to build the system of union catalogue and computer retrieval; in nineteen eighties, focus on the development of on-line retrieval system, regional network and decision making support; in nineteen nineties, focus on the development of internet information service, electronic publishing, content development technology. ${ }^{[3]}$

Traditional information management methods are to mark the outside characteristics to provide search points, or to carry through classification management, or to select subject headings according to the main objects of document content. Above methods played perfect roles in the years of organizing documents by manual mode, but are powerless in the era of information explosion.

In nineteen eighties, the famous information scientist 
Rooks put forwards the concept of cognition map to provide spatial knowledge. American information scientist H·Small put forwards the concept of network map to describe the cause and effect of important discovery. The cognition map and network map represents the development direction of knowledge management.

\section{Geographical Information Technology and Its Functions}

More than 80 percent of human activities are related to geographical location, and geographical information is the integration basis for other socioeconomic data. The deepening of knowledge management requires to build comprehensive resource management system based on GIS, and to improve the capacity of knowledge management and discovery.

\section{A Geographical Information}

Geographical information is the general term of numbers, text, images and graphics to express the quantity, quality, distribution characteristics, internal relationship and change rule of geographical elements. It includes two types, one is spatial location information; the other is attribute information.

Geographical information provides basic and special information related to geographical location, and are correlative with population, resources, environment, socioeconomic development and everyday life.

Geographical information is the record of spatial cognition, the tool for communication, the representation of world view and the guide of future ways. Geographical information is the indispensable media for human to cognize world and to make use of naturalness.

\section{B Geographical Information Technology}

Geographical Information System ( GIS ) is the management technology about geographical spatial information, which has the basic functions of data input, storage, edit, display, inquiry and analysis.

GIS is the ideal platform for spatial data transaction and analysis, is the "majordomo" of spatial information and the common position basis, and is the important technology platform for knowledge discovery and management about spatial information.

1) Map and text integration management is the basic characteristic of GIS. GIS may provide the visual presentation of topography, physiognomy and ground object, offer the common platform for information integration based on spatial location, and support the query from map to text or from text to map.

2) Analysis is the basic function of GIS. Aggregate analysis such as unite, trim and intersect may produce new spatial element. Topology analysis about point, line and polygon may widely used in path analysis, relation analysis and route optimization. Buffer analysis may calculate the number of distributed objects around point and line.

3) Mathematical statistics is the extension of GIS on spatial analysis. The calculation of geometric center and function center, and the evaluation of center moving path and deviation ratio may analyze the changing law of spatial center. The evaluation of compactness or dispersion ratio is to analyze the spatial shape of city. The evaluation of radial shape index is to analyze the internal relation among city.

4) Spatial information mining is the elevation of GIS on the knowledge management. Decision-making system includes data warehouse, OLAP (On-Line Analytical Processing), data mining, models and expert system. Data warehouse may carry out the data storage and integration according to decision theme. OLAP may carry out multiple-dimension data analysis. Data mining may acquire knowledge from database and data warehouse. Models may help to carry out quantitative analysis, and expert system makes use of knowledge to carry out qualitative analysis.

\section{Development of the Knowledge Management Based} on GIS

\section{A Cognition Map}

The basic application of GIS in knowledge management is to associate all sorts of information with spatial location, carry out visual management, and help to form cognition map, which reveals the spatial relation and variation of knowledge.

The geometric analysis of GIS may help to acquire new knowledge and make knowledge management optimize spatially. Mathematical statistics may help to discover the not easy directly observed internal laws from large amount of data and explain the spatial status and evolution with digital information, which adds the accuracy and comparability of spatial evaluation.

Data mining based on GIS is the effective means to make full use of spatial information, which may automatically, rapidly, effectively discover useful information and connotative knowledge and make the limited data to limitless knowledge. ${ }^{[4]}$

\section{B Network Map}

Internet is the ideal technology platform to carry out resource sharing and collaborative work. Internet carries out the connection of computer hardware, and Web carries out the connection of web pages, which provides the communication of e-mail and web browsing. Internet makes the fully sharing possible of computer resource, storage resource, communication resource, software resource, information resource and knowledge resource. ${ }^{[5]}$

The knowledge chain will break through the regional and institutional limitations, and form the resource pooling and sharing for the distributed data, which is just the network map for knowledge management.

Nowadays Internet of things developed quickly. Internet carries out the connection of people with people, and Internet of things carries out the connection of things with things. On the basis of Internet, Internet of things use the technology of RFID and wireless communication, connect any things in the word with Internet to exchange information, which will bring great influence to knowledge management. ${ }^{[5]}$.

\section{National Conditions Map}

Geographical national conditions include territory area, region division, landform characteristic, road network, rivers and lakes, land use, urban layout and expansion, which consist of basic geographical information, geographical statistics information and geographical evolution information. 
Geographical national conditions monitoring is to make full use of geographical information resource and modern surveying technology, select important elements to carry out dynamic monitoring, improve the data integration and analysis level to reveal the spatial distribution and variation laws of social economic and natural environment, and provide authoritative, objective and accurate basic national conditions to improve scientific decision-making. ${ }^{[6]}$

Geographical national conditions monitoring has been put forward as an important task, which will greatly improve the knowledge discovery and management about spatial information. National conditions map will improve the cognition map and network map to a new level.

\section{Conclusion}

Knowledge management is the effective means to collect, process, manage, transmit and make use of information in this information explosion age. The application of geographical information technology will give the fully new concept for knowledge management, which will improve the deepness of spatial data management and mining and using, and expand the extent of information distribution and sharing. The development and application of new technology will make the knowledge innovation rapidly increase.

\section{References}

[1] Al Gore. Digital Earth-Understanding the Human Earth of 21st Century [J]. Geography Research, 1998(Supplement), pp.1-2.

[2] Xia Jinhua, Jin Xin. Knowledge Management [M]. Bei Jing, China Machine Press, 2003, pp.48-57.

[3] Su Xinning, Deng Sanhong. Enterprise Knowledge Management System[M]. Bei Jing, China Science Press, 2004, pp.95-121

[4] Li Deren, Wang Shuliang. On Spatial Data Mining and Knowledge Discovery [J]. Journal of Wuhan University (inf.sci), 2001(6), pp.1-6.

[5] Li Deren, Gong Jianya. From Digital Earth to the Wisdom of Earth [J]. Journal of Wuhan University (inf.sci), 2010(2), pp.1-4.

[6] Xu Deming. Take on the New Mission of Geographical National Conditions Monitoring [N]. China Surveying and mapping news,2010-12-26(1). 